\title{
Impact of Ultra-Wideband Antenna Application on Underground Object Detection
}

\author{
Dr. Zahid Ali ${ }^{1}$, Ghulam Abid ${ }^{1}$, Chandar Kumar ${ }^{1,2}$, Vengas Memon ${ }^{1}$, \& Aysha Siddiqui ${ }^{3}$ \\ ${ }^{1}$ Faculty of Engineering Science and Technology, Indus University, Karachi, Sindh, Pakistan. \\ ${ }^{2}$ Department of Electrical Engineering, DHA Suffa University, Karachi, Sindh, Pakistan. \\ ${ }^{3}$ FAST-National University of Computer and Emerging Sciences, Karachi, Sindh, Pakistan. \\ arain.zahid@indus.edu.pk, ghulamabid@indus.edu.pk, chandar.malhi@yahoo.com, \\ vengas.memon@indus.edu.pk, \& aysha.siddiqui@nu.edu.pk
}

\begin{abstract}
Ground Penetrating Radar (GPR) is efficient method of detecting underground objects. It is a geo-physical technique used to illustrate the subsurface objects by using electromagnetic waves. The electromagnetic pulses are transmitted and received by the ultra-wideband (UWB) antenna in the GPR module for the identification of hidden objects. The problem in detection of buried objects occurs due to antenna reflections; which is resolved by altering the antenna structure. The reformed designed structure practically roofed complete Ultra-wideband bandwidth. The initiated antenna structure and optimization technique is designed by simulation software termed 3D CST. The tested of antenna performance factors such as Reflection coefficient; Voltage Standing Wave Ratio (VSWR) and transient behavior through simulation software. The prototypes of the conventional and initiated reformed antenna have designed and tested projected for its directional pattern as well as return loss
\end{abstract}

Key words : Ground Penetrating Radar (GPR), the ultrawideband (UWB) antenna, CST Microwave Studio

\section{INTRODUCTION}

GPR makes the use of electromagnetic waves for determining the objects and their properties in Subsurface. There are three main parts of the GPR system, i.e., UWB antenna, control unit and the power source. The UWB antenna of the GPR system transmits a short pulse of an electromagnetic wave in the ground then reflected from the hidden object or medium with different dielectric properties. The reflected signal is then sensed by the receiving antenna and is displayed on the screen in real time. For later processing and interpretation, the reflected data can also be stored in the memory [1] Antenna is a very important component of the GPR system, which emits and collects short pulses for detection of buried objects inside the earth. UWB antennas are used in the GPR systems [2]. There are many types of antenna. One of the types is Monopole antenna. It is placed on the ground and works with a double wire in a way that is connected to the antenna and the other is connected to ground plane. It has omnidirectional Radiation pattern. They have the advantages of being smaller in size and are cost effective. Substrate based planar antennas have lower efficiency [3]. The second type is dipole antenna. It is used as an UWB antenna for GPR applications. They are powered by feeding of different natures such as micro strip, coplanar and with coaxial. The direction of radiation of the dipole antenna shows higher gain and good symmetry [3]. TEM horn antennas are frequently used horn antenna that has UWB characteristics. These antennas are complex, made by reducing the two sides of the horns. They have small distortions and good radiation pattern [3]. Tapered Slot Antennas (Vivaldi antennas) are used for the application of UWB. This antenna has advantages of being easy to design, simple structure, small size, and inexpensive design [4]. The UWB antenna is designed for several applications including radar and communication according to their radiating characteristics [2]. UWB $4 \times 1$ is modified Vivaldi antenna array for high gain and good impedance matching [5]. The antipodal Vivaldi antenna for UWB application's range is 3.1 to $10.6 \mathrm{GHz}$ [6]. Some other TEM antenna has demonstrated and optimized with very low latetime ringing for detecting buried objects [7]. The performance of the Vivaldi antenna is increased by corrugating the ending of the antenna flares so that the backward current can be reduced [8]. This antenna has significant gain and linear polarization and can be made to correspond to a constant gain in relation to the frequency [9]. VSWR, reflection coefficient and dispersion in the lateringing needs improvement for batter performance of the antenna [10]. in [11], for $\mathrm{GHz}$ frequency range application a low cost based high gain microstrip array antennas with advance inputs methods and dielectric substrate is disused. In this research efficiency of the antenna improved by upgrading the structure of antenna, reflections are minimized for improving the performance of the antenna for GPR applications.

Section 2 discusses the methodology, the model design of Antenna is described in 3, Section 4 provides results and discussions and finally conclusion is given.

\section{DESIGN AND CONFIGURATION OF ANTENNA}

This antenna can be designed in a single layered structure called the tapered Vivaldi antenna that provides sufficient reflection and small dimensions. The different size of width and length of the taper opening can bring effects to Voltage Standing Wave Ratio and S11. Tapered slot antennas usually have high directivity, wide bandwidth, and can create a symmetric radiation pattern. It is characterized by an exponential flare shape. At the flare beginning, the width defines the maximum frequency, and at the mouth of the flare, the width defines a minimum frequency. It is powered from the slot line. In this design we have used a discrete slot 
for powering the slot. UWB is defined in terms of relative bandwidth.

$$
2(\mathrm{fH}-\mathrm{fL}) /(\mathrm{fH}+\mathrm{fL})>0.2
$$

\section{Radiating curve equation}

$$
\begin{aligned}
& \mathrm{u}(\mathrm{t})=\mathrm{t} \\
& \mathrm{v}(\mathrm{t})= \pm \mathrm{S} \times \exp ^{\wedge}(\mathrm{r} \times \mathrm{t}) \\
& \mathrm{w}(\mathrm{t})=0
\end{aligned}
$$

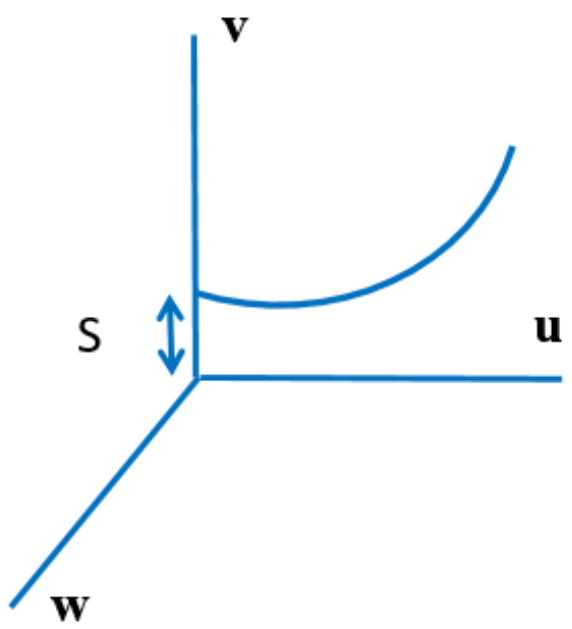

Figure 2: Design of Taper using equation

Where $\mathrm{t}$ and $\mathrm{v}$ are in same unit, $\mathrm{v}$ is in the substrate axial direction and $\mathrm{t}$ is in the horizontal direction as shown in Figure 02. It is clear that for propagation of the EM wave in the free space, the characteristic impedance, and the slot measurement variable in correspondence with the position of the discrete port should be appropriately selected. The characteristic impedance of the given antenna is calculated according to:

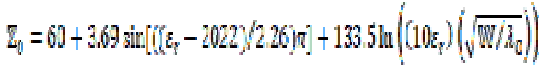

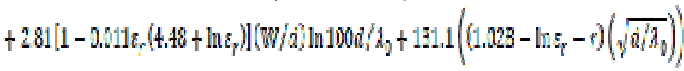



Where,

$\lambda_{0}=$ operating wavelength

$\mathrm{W}=$ slot line width

$\mathrm{d}=$ dielectric substrate thickness

$\mathrm{Z}_{\mathrm{o}}=$ impedance of the slot line $(\Omega)$

$\varepsilon_{\mathrm{r}}=$ relative permittivity of the dielectric

Therefore, these designs have been implemented in CST using the above mentioned equations and design parameters are given in table 01 below:
Table 1: Design parameters for the conventional Vivaldi antenna

\begin{tabular}{|l|c|}
\hline $\begin{array}{l}\text { Resonant } \\
\text { Frequency }\end{array}$ & $\mathrm{F}=36.89 \mathrm{GHz}$ \\
\hline Width of Substrate & $\mathrm{W}=6.5 \mathrm{~mm}$ \\
\hline Substrate material & RogersRT5880(lossy) \\
\hline $\begin{array}{l}\text { Thickness of } \\
\text { Substrate }\end{array}$ & $\mathrm{H}=0.2 \mathrm{~mm}$ \\
\hline $\begin{array}{l}\text { Relative } \\
\text { Permittivity }\end{array}$ & $\varepsilon_{\mathrm{r}}=2.5$ \\
\hline Loss tangent & 0.0009 \\
\hline Taper Rate & $\mathrm{R}=1.0$ \\
\hline Slot line Width & $\mathrm{Sw}=0.1 \mathrm{~mm}$ \\
\hline Length of slot & $\mathrm{Sl}=2.7 \mathrm{~mm}$ \\
\hline Taper length & $\mathrm{Tl}=6.6 \mathrm{~mm}$ \\
\hline
\end{tabular}

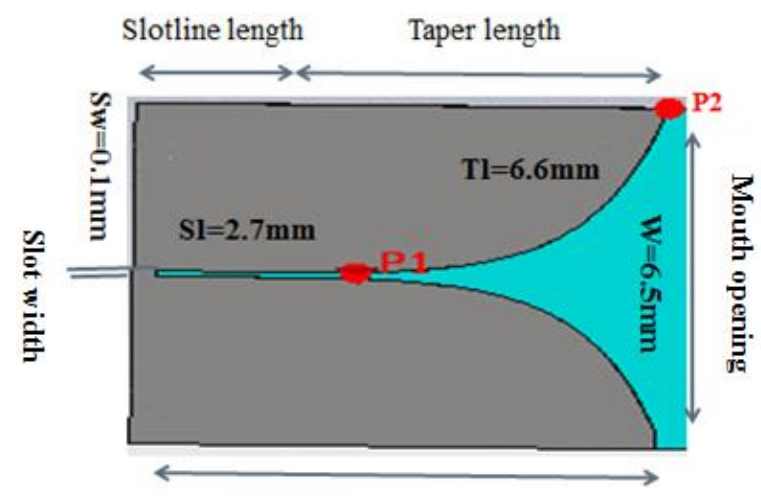

Antenna length

Figure 3: Conventional antenna

\section{DESIGN AND MODELING OF OPTIMIZED VIVALDI ANTENNA}

It is important to consider for the GPR application is the characteristic of the Vivaldi antenna with respect to time domain and the most important thing is that both reflection from antenna and ringing should be minimal. The Vivaldi antenna is designed to reduce the ringing and reflection from the antenna assembly. We can modify the Vivaldi antenna by changing the discrete port position to increase the frequency of the resonance. The discrete connection of the optimized Vivaldi antenna is shown in Figure 4. 


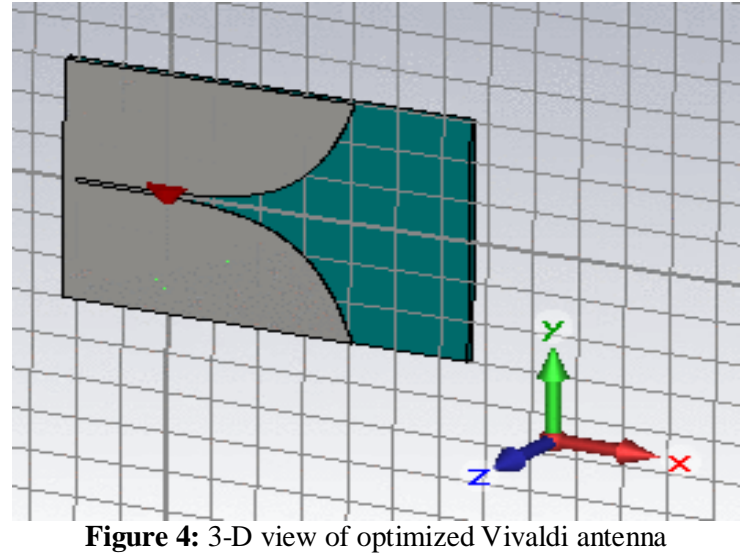

The GPR antenna is located on the surface and sends short impulses through a deep broad band connection to the subsurface, the part of the signal is reflected from the surface and the rest goes to the ground where it reflects back once when it hits the scatterer beneath the surface. However, the antenna focuses on coming reflected waves and are processed and stored in the system memory. During this comparative analysis of the optimized Vivaldi antenna, a single UWB bi static mode is often used to transpose waveforms and receive a reflected signal from the object and the earth. The Vivaldi antenna is used to range the frequency range of the GPR environment from $30.582 \mathrm{GHz}$ to $41.712 \mathrm{GHz}$ which is capable of radiating the RC2 pulse of 0.054 ns. Optimized Vivaldi antenna is shown with the earth, with relative permittivity ( $\varepsilon r$ ) of 5 . The antenna is placed at a distance of $3 \lambda_{0}(\mathrm{~mm})$ from the earth. The boundary condition of the open (add space) is set in all directions. The GPR environment can be shown in Figure 05 below:

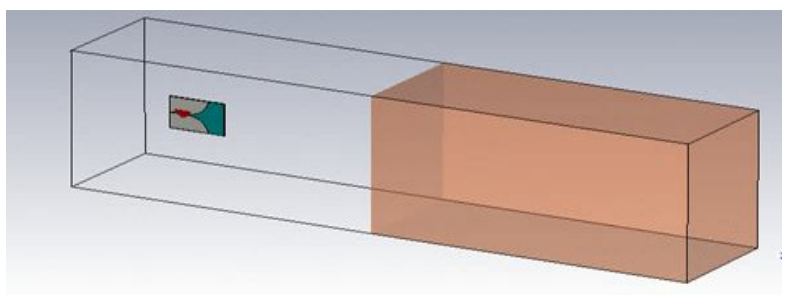

Figure 5: 3D view of Modeling of optimized Vivaldi antenna with earth

Here, the Vivaldi antenna is modeled with earth and scatterer. The earth with relative permittivity (cr) of 5 is selected for modeling. The antenna is set at a distance of $3 \lambda_{\mathrm{o}}$ from the earth. In earth, a Perfect Electric Conductor (PEC) the scatterer is at a depth of $6 \lambda \mathrm{e}$. This design was made to identify the scatterer (object) under the surface of earth with the enhanced Vivaldi antenna. The general GPR situation is shown in Figure 06. In all different directions of the computing domain; an open (adding space) boundary condition is applied.

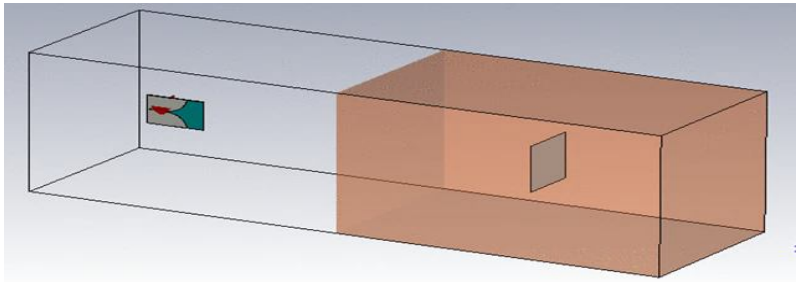

Figure 6: 3D view of Modeling of optimized Vivaldi antenna with earth and scatterer

Table 2: Design Parameters for the optimized Vivaldi antenna

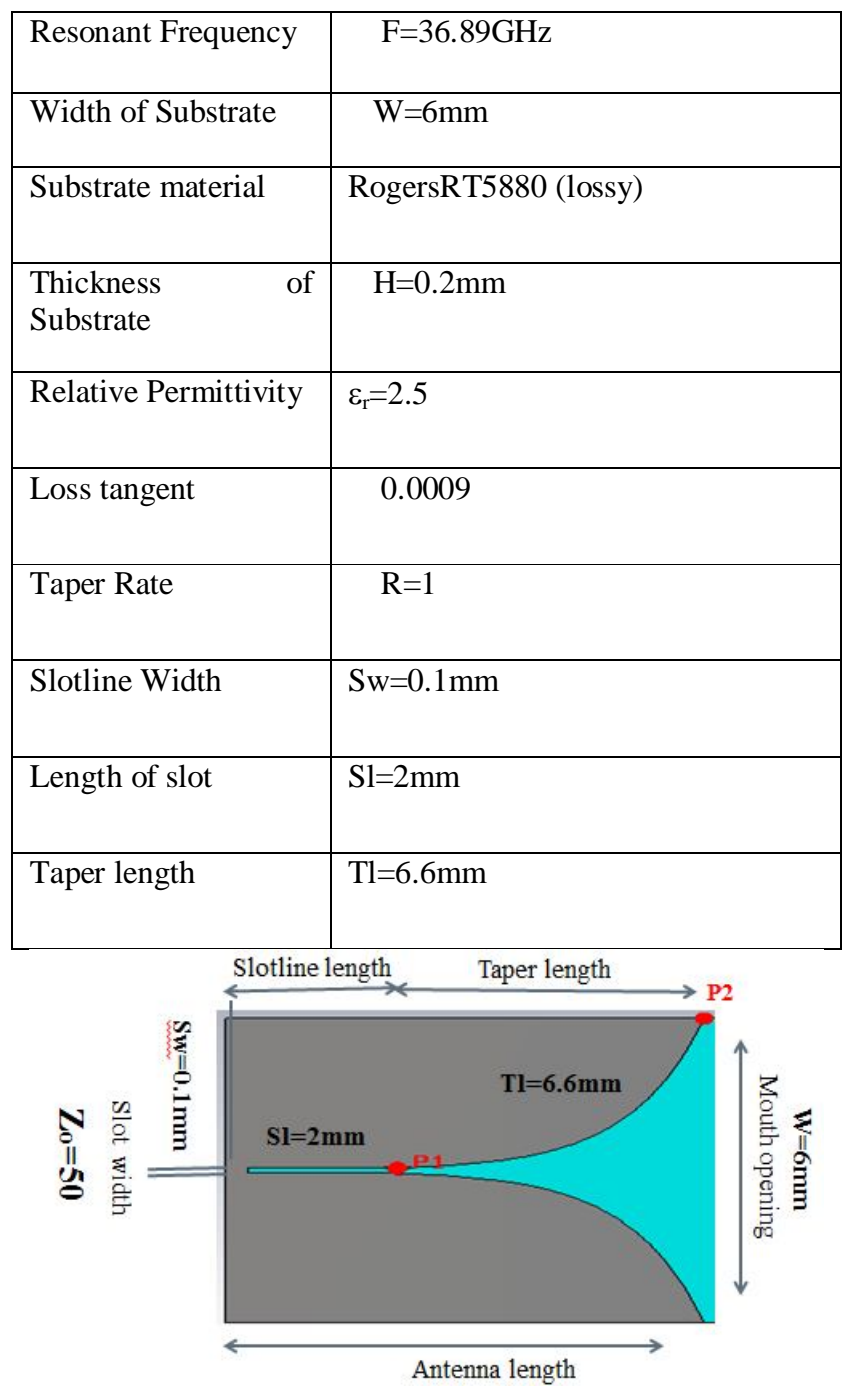

Figure 7: Optimized Vivaldi antenna

\section{RESULTS AND DISCUSSIONS}

This part discusses the results of the conventional and optimized Ultra Wideband (UWB) antenna for GPR applications in detail. The results include the response of the antenna such as Reflection coefficient; Voltage Standing Wave Ratio (VSWR) and transient behavior, the simulation results of the conventional and optimized antennas are also compared. The optimized antennas are then tested in the GPR environment. 


\subsection{OPTIMIZED VIVALDI ANTENNA}

\subsubsection{Input signal and Reflected signal}

Optimized Vivaldi antenna has been designed in CST Microwave studio 2014 software. The RC2 pulse being an input signal has $0.054 \mathrm{nsec}$ width that is used for the simulation of time domain. The port of the outpt signal shows reflection from the antenna. The modified Vivaldi antenna has low reflection and ringing.

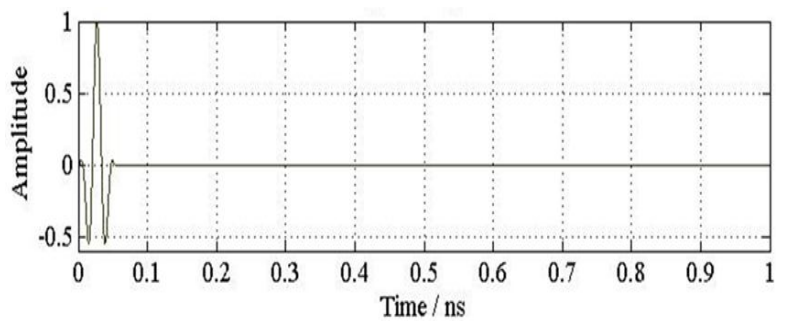

Figure 09: Input signal

Figure 10: Reflected signal of Vivaldi antenna

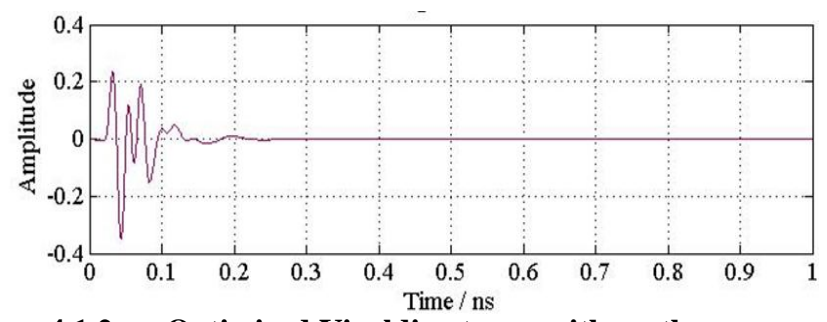

\subsubsection{Optimized Vivaldi antenna with earth}

In this section, the Vivaldi antenna is designed with earth has relative permittivity ( $\mathrm{cr}$ ) of 2.5. The antenna is placed at a distance of $3 \lambda \mathrm{o}(\mathrm{mm})$ from the earth. The signal radiated by the antenna is empty, small portion of the signal is reflected back from the outside of the earth, and the other part is transferred to the earth. Getting a reflected sign from the surface of the earth and on the discrete port, the quantity of signals is stored at the discrete port while the output signal contains a reflected signal from the earth and the antenna itself from the earth. The speed of the signal transmitted within the earth is low from the signal traveling in an empty space due to the properties of the earth.

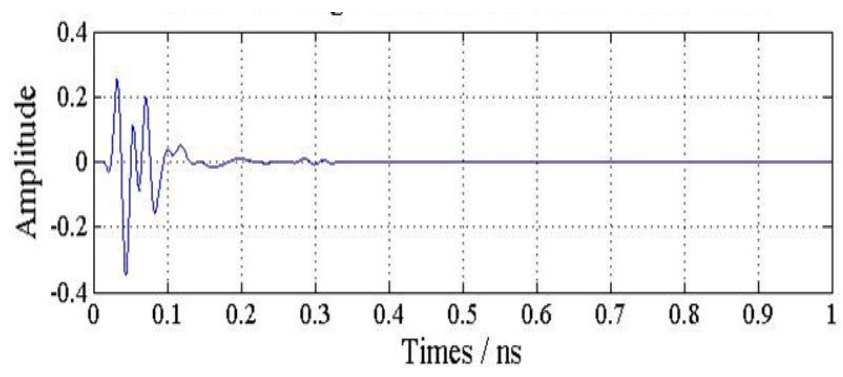

Figure 11: Reflected signal of Vivaldi antenna and earth

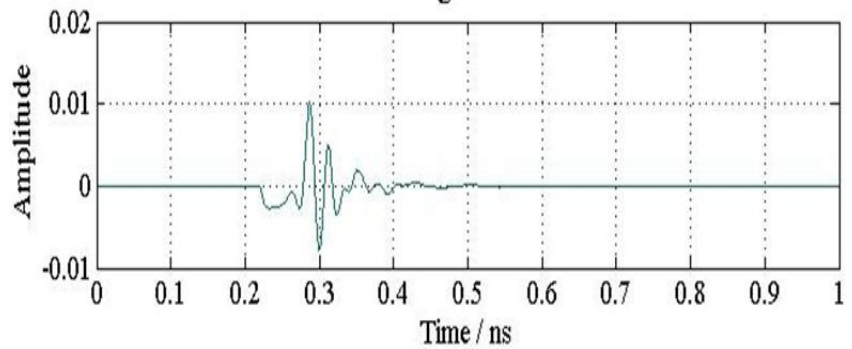

Figure 12: Reflected signal from earth

\subsubsection{Optimized Vivaldi antenna with the Earth and the Scatterer}

Similarly, typical earth penetrating radar (GPR) environment in this region is shown and simulated. The main goal is to detect objects buried in the earth's layers using Vivaldi antenna. The depth of perfect electric conductor (PEC) scatterer in the ground is $6 \lambda \mathrm{e}$. In both cases, the antenna is located above the earth in a free space at a height of $3 \lambda_{0}$. Antenna radiates the signal to the earth. This signal is bounced back from scatterer and earth. A signal that is scattered from the scatterer and earth, is absorbed by given antenna and is kept on the discrete port. To find the returned signal from the PEC scatterer, the output signal of the port is subtracted from the reflected earth and antenna signals, as shown in Figures 14 and 15. This gives a picture of the radiation of an electric field from an antenna to a scatterer inside the earth.
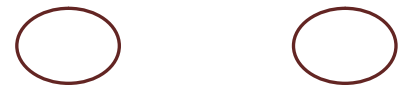

Figure 13: Reflected signal of Vivaldi antenna, earth and scatterer

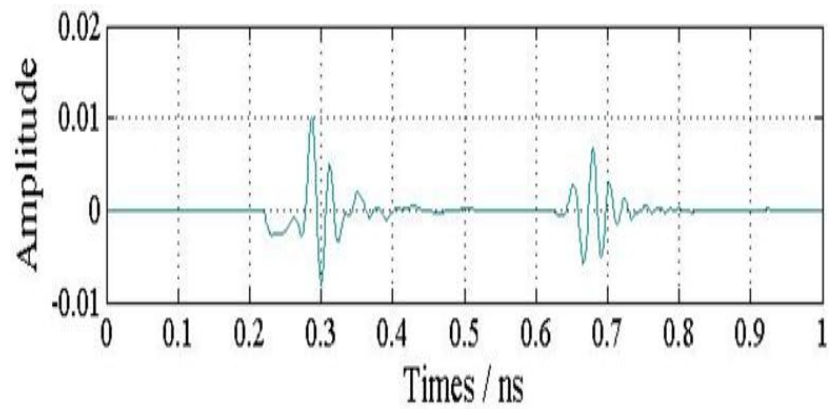

Figure 14: Reflected signal from earth and scatterer

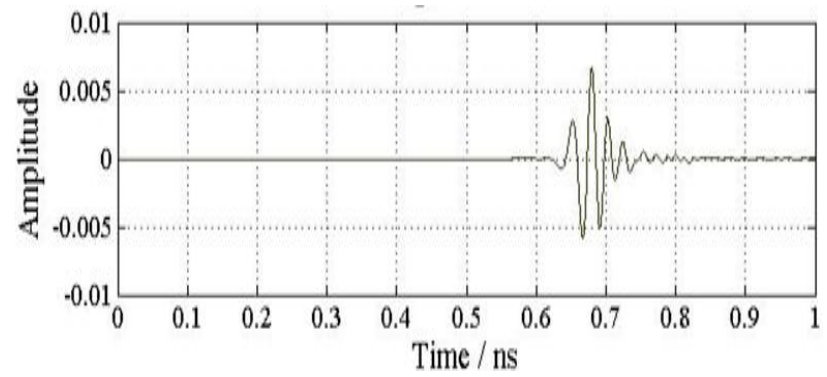

Figure 15: Reflected signal from scatterer 


\subsection{COMPARISON BETWEEN CONVENTIONAL AND OPTIMIZED VIVALDI ANTENNA}

\subsubsection{Time domain reflected signal}

The comparison graph clearly depicts that the internal reflection of the conventional antenna is on the higher side than the optimized Vivaldi Antenna. Due to the higher level of internal reflection, information about a buried underground object is not possible with a conventional antenna. For better performance, it is necessary to improve impedance matching so that internal reflection and late time ringing can be minimized. By changing the value of S1 to high level from 2 to 2.7 more reflections occurs of conventional antenna and when $\mathrm{Sl}$ is 2, minimum reflections occurs as compare to conventional antenna.

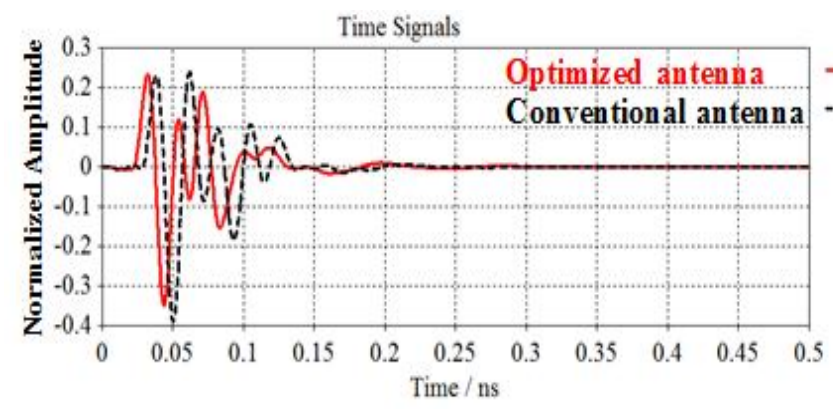

Figure 16: Comparison of reflected signal of conventional Vivaldi antenna

\subsubsection{S-parameter}

The scattering matrix (S-Parameters or S-Matrix) is a mathematical representation that shows amount of power lost in the system due to the mismatched impedances through a multiport network. It displays the ratio of reflected power to reference power in $(\mathrm{dB})$. By changing the value of Sub W to high level from 6 to 6.5 the dip of S-parameter shifts to left $(-12 \mathrm{~dB})$ of conventional antenna and when Sub W is selected as 6, the dip of S-parameter is $(-18.5 \mathrm{~dB})$ of optimized Vivaldi antenna that shows better results.

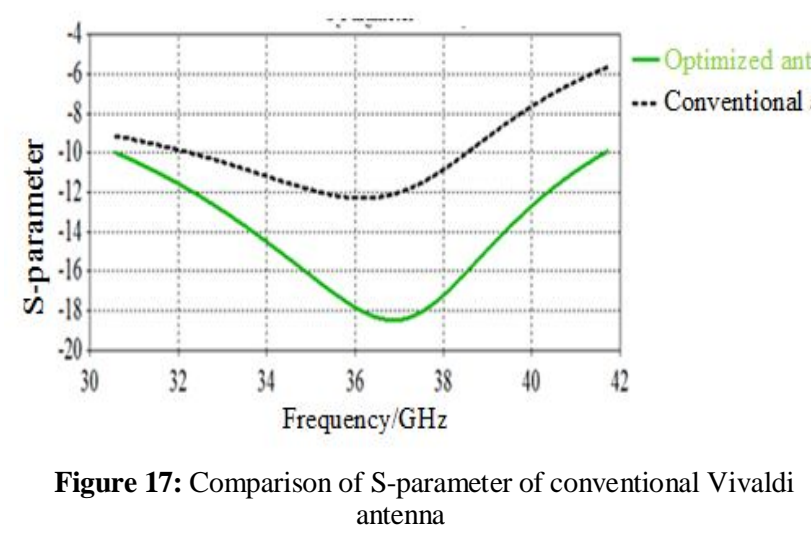

\subsection{VOLTAGE STANDING WAVE RATIO}

The voltage standing wave ratio VSWR describes how well a system is matched (system linearly).In order to obtain the maximum power that is radiated from antenna, ppreferably the measured ratio should be less than 1 . The ratio of the highest to the lowest voltage for the standing wave is VSWR. From the VSWR graph as given below, when $\mathrm{Tl}$ is 6 the dip of VSWR shift to left $(1.6 \mathrm{~dB})$ and by changing value of $\mathrm{Tl}$ is 6.6 , the dip of VSWR $(1.2 \mathrm{~dB})$ gives better results.

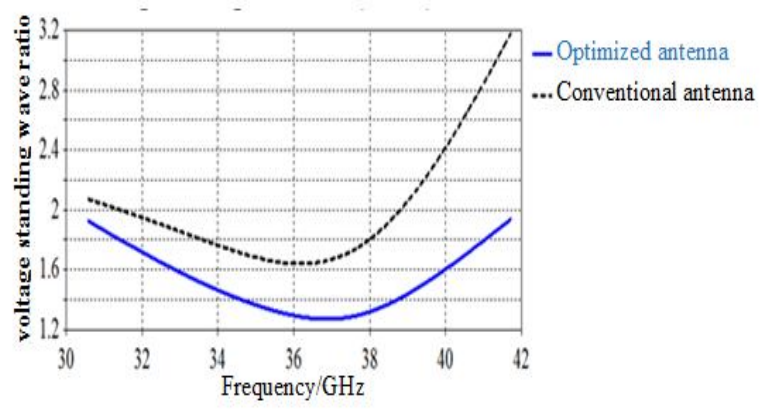

Figure 18: Comparison of VSWR of conventional Vivaldi antenna

\section{CONCLUSION}

In this article the modified Vivaldi antenna is proposed. The structure of new designed antenna is termed as optimized Vivaldi antenna and analyzed under specified parameters. The evaluation of conventional antenna and optimized Vivaldi in term of Reflection coefficient; Voltage Standing Wave Ratio (VSWR) and transient behavior has been argued. It has been observed that proposed optimized Vivaldi antenna has shaped increased directivity at 30.582 $\mathrm{GHz}$ to $41.712 \mathrm{GHz}$ of frequency range.

Furthermore, the normalized amplitude of the reflected signal from the optimized antenna is 0.006 , which is $35 \%$ less than the conventional antenna and the S-parameter of the optimized antenna is 1.2 for the frequency of operation, which is $75 \%$ better than the conventional antenna. Therefore optimized Vivaldi antenna is also significant for underground object detection and Radar application.

\section{REFERENCES}

http://bchazmat.com/bchazmat-services/groundpenetrating-radar-gpr/. [accessed on January.15, 2017].

Weisbeck, W., Adamiuk, G. and Sturm, C. (2009), "basic properties and design principles of UWB Antennas" Proceedings of the IEEE, V.97, No.2, pp.372-385.

Hertel, I., and Strycek, M. (2007), "UWB antennas for ground penetrating radar applications" Applied Electromagnetics and Communications, ICECom 2007,19th International Conference on IEEE, pp.14.

Shao, Wenyi, and Ryan S. Adams, (2013), "Two antipodal Vivaldi antennas and antenna array for microwave early breast cancer detection" Microwave and Optical Technology Letters 55.3, pp. $670-674$.

Chakrabarti, N., Kalra, S., Saxena, S. and Tripathi, M.R. (2016), "Ultra-wideband antenna for a ground penetrating radar" Thirteenth International Conference on, IEEE, Wireless and Optical Communications Networks (WOCN), pp.1-6. 
[6] Moosazadeh, M., and Kharkov sky, S. (2015), "Development of the antipodal Vivaldi antenna for detection of cracks inside concrete members" Microwave and Optical Technology Letters, V.57, No.7, pp.1573-1578.

[7] Jamali, A. A., and Marklein, R. (2011), "Design and Optimization of Ultra-Wideband TEM Horn Antenna for GPR Applications" General Assembly and Scientific Symposium, 2011 XXXth URSI, pp. 1-4.

[8] Maksimovitch, Y., Mikhnev, V. and Vainikainen, P., (2009), "Characterization of tapered-slot antenna designs for subsurface radar applications "Proceeding of 3rd European Conference on Antennas and Propagation, Eu CAP, pp. 28112813.

[9] Gibson, P.J., (1979), "The Vivaldi aerial" Microwave Conference, 9 th European IEEE. pp. 101-105.
[10] Liu, Z., Zhu, Y. and Zhou, J. (2016), "Improved design of Vivaldi antenna with loading resistance for wideband applications" Microwave and Millimeter Wave Technology (ICMMT), IEEE International Conference, V.1, pp. 286-289.

[11] Md. Tanvir Ishtaique-ul Huque, et.al, "Design and Performance Analysis of Microstrip Array Antennas with Optimum Parameters for X-band Applications", (IJACSA) International Journal of Advanced Computer Science and Applications, Vol. 2, No. 4, 2011. 